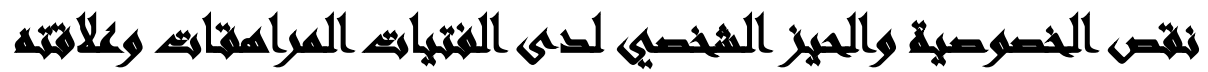

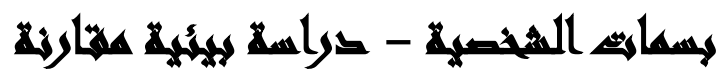

$[r \cdot]$

$$
\begin{aligned}
& \text { ليلى كرم الدين(')- حسام الدين البرومبلي(؟)- أحمد فخري هاني(") } \\
& \text { منى عطيه عبد الموجود لاشين الرومين }
\end{aligned}
$$

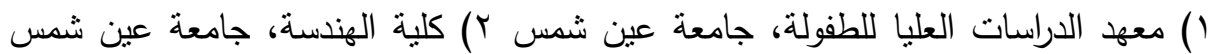

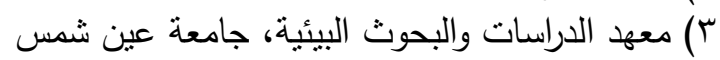

\section{المستخليه}

اهتمت هذه الدراسة بنقص الخصوصية والحيز الثخصي لما لهما من تاثثير على سمات الثخصية. وهدفت الدراسة إلى التعرف على وجود علاقة بين نقص الخصوصية ولتية وانتهاك

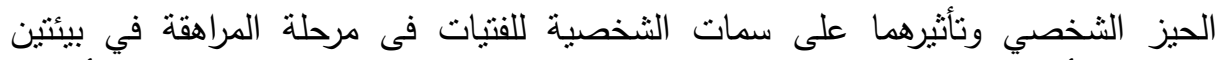

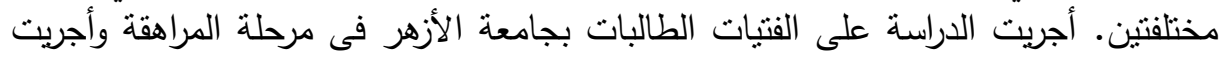

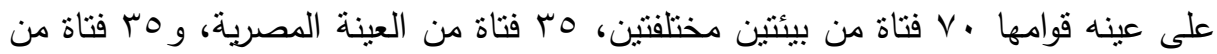

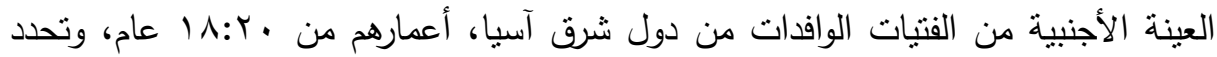

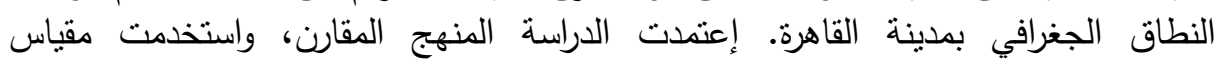
الخصوصية ومقياس الحيز الثخصي؛ ومقياس البيئة الفيزيقية - من إعداد الباحثون، وقائمة

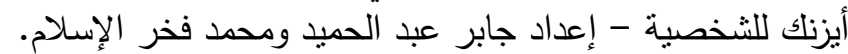

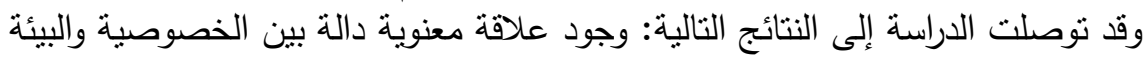

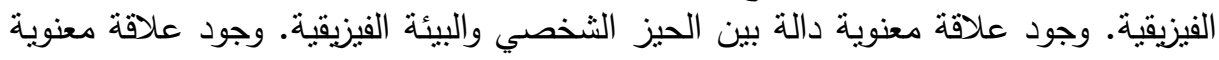

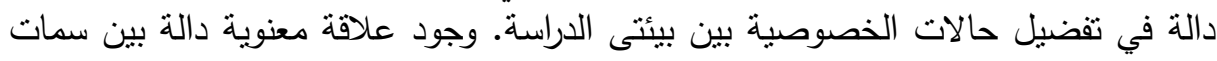
الثخصية ونقص الخصوصية. وجود علاقة معنوية دالة بين سمات الثخصية وانتهاك الحيز

الثخصي.

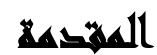

تزايد الاهتمام بموضوع البيئة منذ منتصف الستينات من القرن الحالي، وذلك الاهتمام يرجع لأهمية تأثنر البيئة على الانسان وسلوكياته وتتدر الدراسات العربية التي نقوم بدراسة لئه

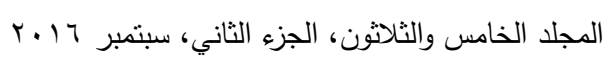


تأثثر نقص الخصوصية والحيز الشخصى على سمات الثخصية للفتيات وهذا ما تسنوفيه هذه

Engineering \& Environmental قد برز علم النفس الهندسي والبيئي عsychology لصالح الأفراد والجماعات، فهو يهنم ـ كما جاء في مجلة الملخصات السيكولوجية Psychological Abstracts - بدراسة العوامل في المجال الهنسي، والتصميمات فولاء والتخطيط البيئي والمجمعي، أي أن اهتمام علم النفس البيئي هو التفاعل بين الانسان والبيئة المحيطة به.وقد كان علم النفس العمراني هو الباعث لظهور علم النفس البيئي . وهو يؤكد دور الموقف الفيزيقي في استثارة السلوك الإنساني.

وعلى الرغم من أن معظم الاهتمامات قد نركزت حول البيئة الفيزيقية Physical ، فإن

علم النفس البيئي يمتد ليشمل كلا من البيئة الطبيعية Natural ، والمشيدة:Built ، والاجتماعية Social، ويؤكد على أهمية دراسة استجابات الأفراد والجماعات لهذه البيئات، من حيث تأثرهم بها وتأثيرهم فيها.

البيئة هي المجال الذي تحدث فيه الإثارة والتفاعل لكل وحدة حية وهي كل ما يحيط بالإنسان من طبيعةٍ ومجتمعات بشرية ونظم اجتماعية وعلاقات شخصية ـ وهي المؤثر الذي يدفع الكائن إلى الحركة والنشاط والسعي ـ فالتفاعل منواصل بين البيئة والفرد والأخذ والعطاء مستمر ومتلاحق، و دراسات علم النفس البيئى تؤكد على ملاحظة السلوك كما يحدث في إطاره الطبيعي وليس كما يحدث فى مواقف تجريبية محددة.

يتم تعريف الحيز الثخصي personal space بأنه المنطقة أو المساحة المحيطة بجسم الثخص، والتي لا يتداخل فيها الآخرون دون استثارة عدم الراحة، وهى ليست موقعا لئه

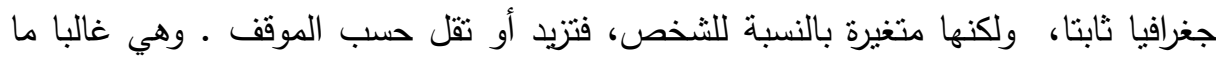

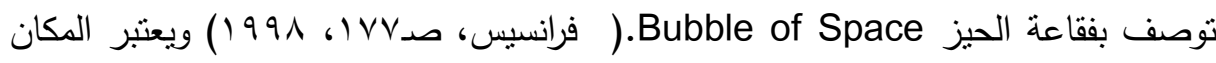
الخصوصي private space حاجة الإنسان لأن ييقى على جزء من حياته الخاصة بعيدا 
عن آذان، أو عيون الآخرين احتباج بشري يحاول بقدر الإمكان أن يحتفظ به دون تدخل من الآخرين، والحاجة للخصوصية، أوالإبقاء شيء للنفس لا يعنى العزلة المكانية. فتحقق الخصوصية النمو وتُشكِل ذات الإنسان فى صورة سليمة فهى من مكونات الهوية الثخصية، وعند المراهقات توجد تحديات خاصة بهذه المرحلة مثل الصراع الداخلي وحدة التعامل وتفرد الرأي وصراع الإستقلالية ونجد عند دراسة الفتيات فى مرحلة تكوين الثخصية

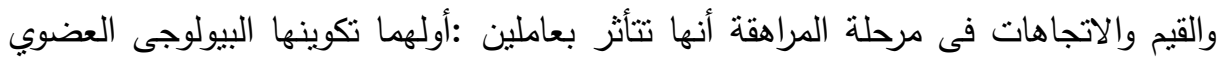

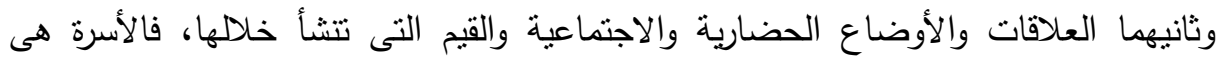
المجال الأول التى تتقل إلى الفتاة كافة المعارف والاتجاهات والقيم التى تسود المجتمع بعد ولاهد تحويلها إلى أساليب عملية لتكوين شخصية مرغوبة من الأسرة والمجتمع، فتتضمن عملية تكوين الثخصية من خلال الأسرة علاقات مختلفة بعضها مرتبط بالحاجات البيولوجية وأساليب إنباعها أو التعبير عنها والبعض الآخر ينعلق بالحاجات النفسية والاجتماعية

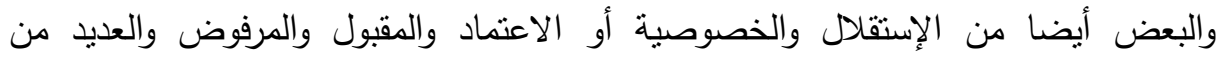
العلاقات التى تحدد النمط العام للشخصية.

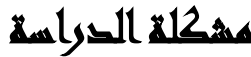

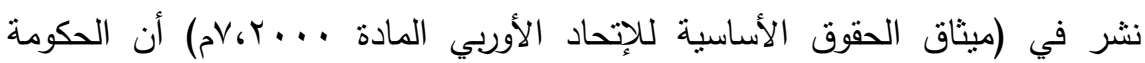

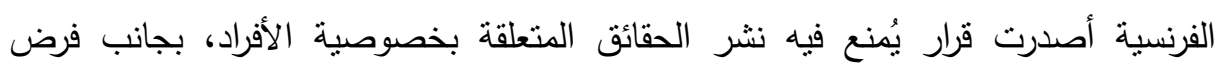

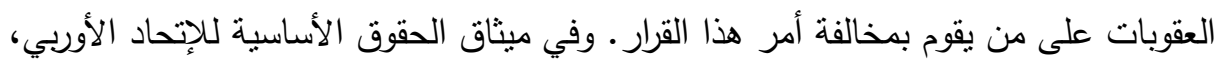

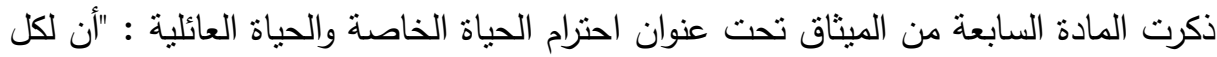

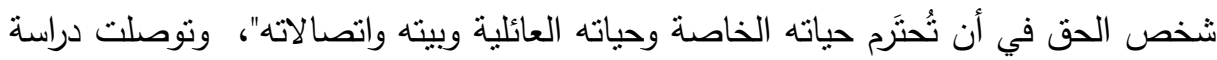

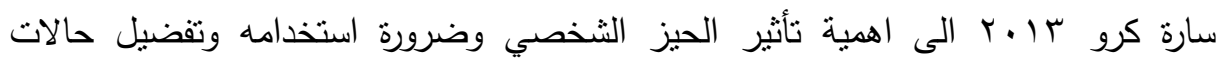

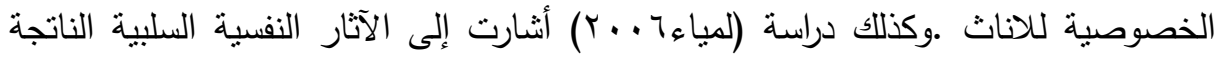

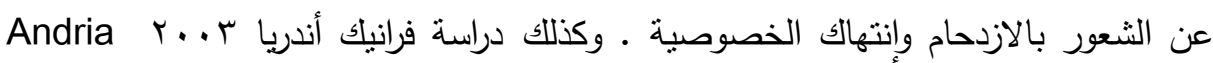

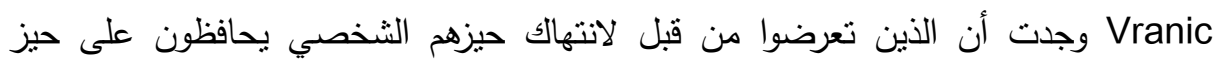
شخصي أكبر بينهم بين الآخرين بطريقة تلقائية. لذلك يرى الباحثون بدراسة تأثنر نقص ئن

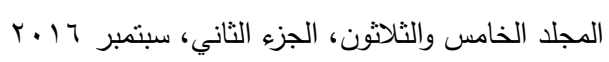


الخصوصية والحيز الثخصي على الفتيات فى مرحلة المراهقة كأحدى المراحل الهامة فى تكوين سمات الثخصية.

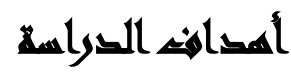

1. التعرف على واقع الخصوصية والحيز الثخصي لدى الفتيات المراهقات بعينة الدراسة. r. التوصل للعلاقة بين الخصوصية والحيز الثخصي وبعض سمات الثخصية في عينة الدراسة. r. التعرف على البيئة الفيزيقة وعلاقتها بالخصوصية و والحيز الثخصي لدى الفتيات بعينة الدراسة .

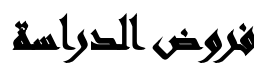

تتطلق الدراسة من فرض أساسي مؤداه: "أنه توجد علاقة ذات دلالة إحصائية بين

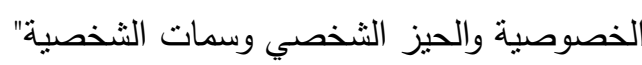
وينطلق من هذا الفرض فروض فرعية: 1-هناك علاقة ذات دلالة إحصائية بين نقص الخصوصية: ورضية وانتهاك الحيز الثخصى لدى الفتبات المراهقات. r-هناك علاقة ذات دلالة إحصائية بين الخصوصية والحيز الثخصي وبعض سمات الثخصية. r-هناك علاقة ذات دلالة إحصائية بين الخصوصية والحيز الثخصي و خصائص البيئة الفيزيقية.

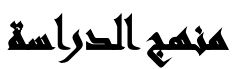

تستخدم الدراسة المنهج المقارن حيث ينيح استخدام المنهج المقارن، التعمق والدقة في الدراسة من خلال المقارنة عند دراسة الظاهرة حيث تبرز أوجه الثبه والاختلاف فيما بين 
ظاهرتين أو مجتمعين مجتمع عربي وآخر اجنبى ـ فحين نفتقر إلى المصادر أو تكون نادرة فان أفضل المناهج هو الدنهج المقارن الذى يربط بين الظواهر بالمقارنة والتحليل.

\section{أهمية الترواسلة}

تهذف الدراسة لتفسير سمات الثخصية لاى الفتيات فى مرحلة المراهة بتأثير نقص/تكامل الخصوصية والحيز الثخصي لايهن. فيقام البحث على الدستوى النظري: فيجد فئد

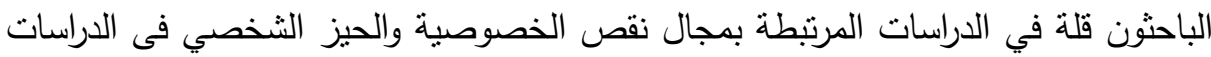

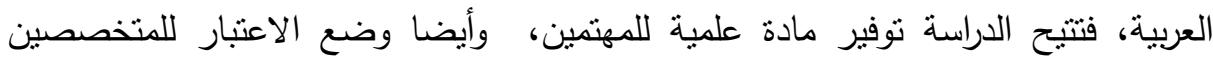

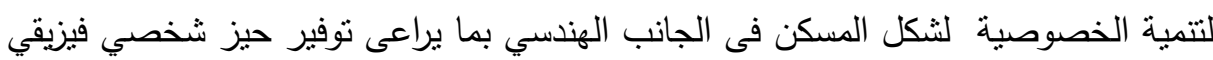

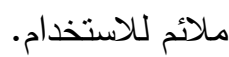

\section{هجالإه الصراسة}

المجال المكانى: للدراسة فى جامعة الأزهر بمحافظة القاهرة ممثلة فى عينة من بعض الطالبات الفتيات من القاهرة والعينة الاجنبية من الوافدات من دول شرق آسيا. المجال البشري: للاراسة عينة عشوائية تكونت من • م مفردة من الفنيات فى مرحلة

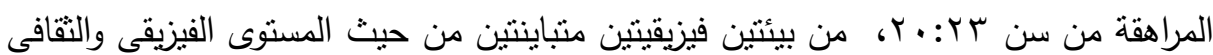

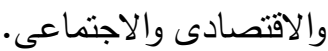

المجال الزمنى: للدراسة بدأت الباحثة فى جمع الاطار النظرى للاراسة ثم العمل على

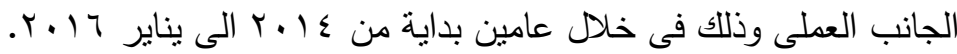

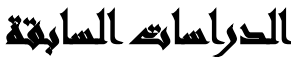

حول الحيز الثخصى تثير دراسة (Serfants Amily 2013) أن التعدى على الحيز الثخصى يرفع من نسبة القلق، وكما وجدت دراسة ( Crowe sarah2013)عند المقارنة بين الذكور والإناث والاعراق المختلفة لأهمية إستخدام الحيز الثخصى، فنتين أن الاناناث

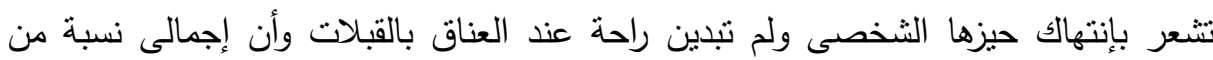

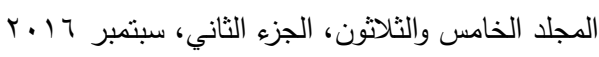


يشعرون بالرضا الكامل للحيز الثخصى الذى يحصلون عليه كانوا 0, • ٪ فقط،، ووجدت ايضا ابعاد مختلفة فى التعددات العرقية فى شعورهم بإنتهاك حيزهم الثخصى ؛ وكانت النتائج

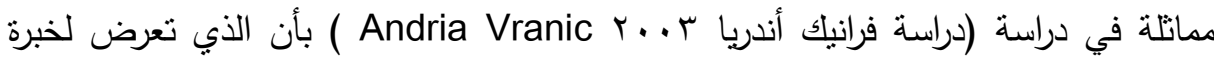

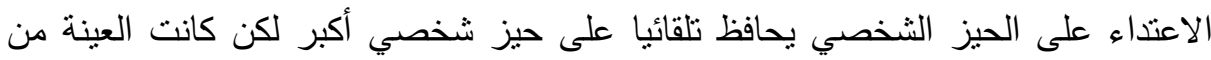
الاطفال صغيرة السن غير عربية ودراسة (ريهام عبد العزيز ج ج. r) وجدت أنه عند تصميم الحيز الثخصى الداخلى، وعندما يتم التعدى عليه فذللك يؤثر على النمو النفسى للإنسان.

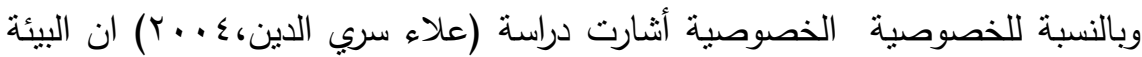
المنخفضة لا توفر الخصوصية، وتزيد من حالات الإنفراد والمجهولية ووالتحفظ والعلاقة الحميمية مع الأصدقاء بدرجة أكبر من البيئة الموفرة للخصوصية، وتوصلت دراسة (Newman russ1999) ان الحد من الخصوصية والسرية يؤدى إلى الإضطراب النفسى والتضارب السلوكى، أما دراسة (زين إحسان r . .r) وجدت تفضيل الإناث للخصوصية فى إلى

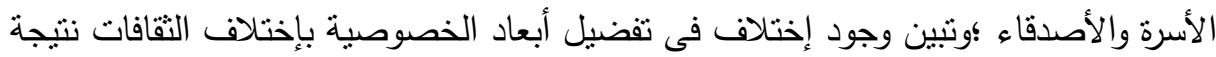

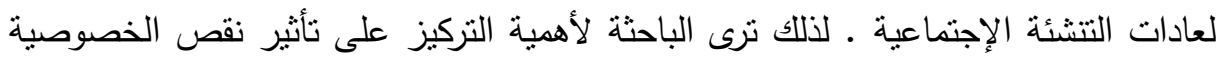

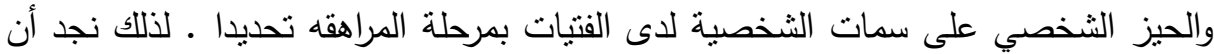
مرحلة المراهقة تمنل أنواع متعددة من الصراعات والتوتر الذي تعيشه المراهقة من خلال الانتقال من الطفولة إلى الرشد،فلذلك تبحث الفتيات لنفسها على مساحة شخصية وحيز لتتعر

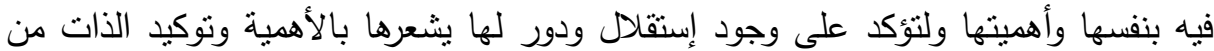

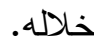

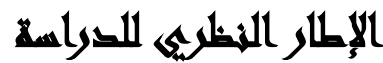

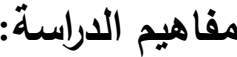

الخصوصية: هي مرادف غربي لتعبير الستر الاجتماعي الموجود في المجتمعات الثرقية، وهو شعور فطري يحتاجه الإنسان على المستوى الثخصي او على مستوى الزوج وزوجته او

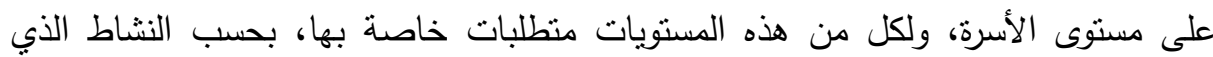

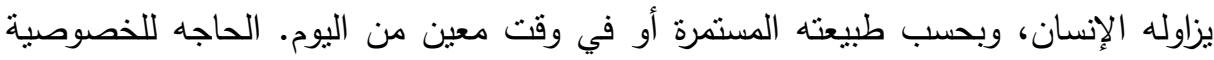
420

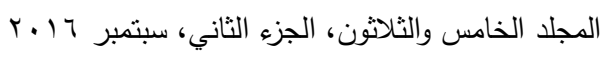


من الحاجات الراسخة في كيان البشرية، وهي بطبيعتها تقوم على النزاهة والكرامة الثخصية، و الخصوصية في سياقات مختلفة تعطي حرية الفكر والضمير، وحرية الوحدة،وحرية التحكم في الجسد، وحرية حماية السمعة، وحرية الحياة العائلية. الحيز الثخصي: لدى علماء النفس البيئى يشير إلى "الحدود المكانية التي تحيط بجسم الشخص ويعتبرها خاصة به هو فقط، لا يجب أن يتعداها الآخرون .و هذه الحدود تحيط بالمرء تتحقق فى المكان وفى النفس فيمثل المنطقة التى تحيط بالفرد من جميع الاتجاهات وتحدد قدرته على تحمل الآخرين أو السماح لهم بالنفاذ إليه والتفاعل معه، يعتبر الحيز

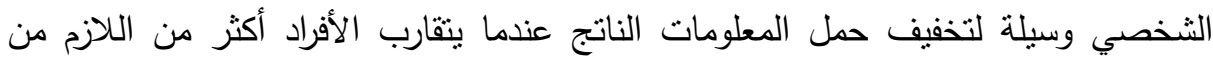

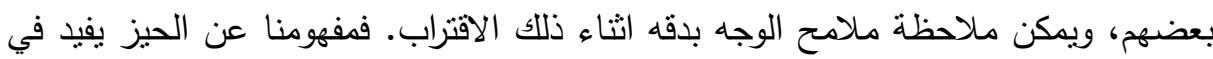
استخدام حدود الأثياء، إذا لم يكن هناك أب حدود فنقوم بصنعها عن طريق إستخدام خطوط لوناه

حالات الخصوصية: حاولت مؤسسة الخصوصية الدولية (privacy international,2014) أن تضفي شيئا من الوضوح فعرفت الخصوصية بأنواعها المختلفة :خصوصية المعلومات مثل هورونل

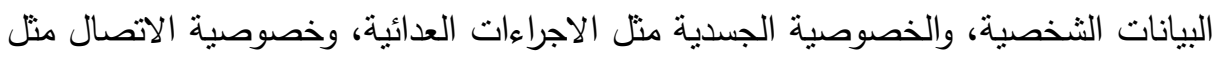
المراقبة، وخصوصية المكان مثل المسكن.

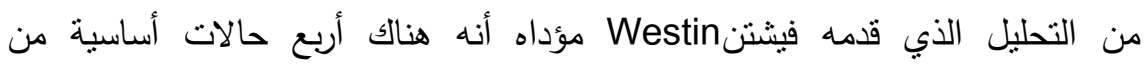

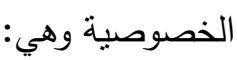
الإنعزال solitude : وتتسم بالفردية وهي حالة من الخصوصية بكون فيها الفرد وحيداً بعيداً عن أي ملاحظة من قبل الآخرين، ومن ثم تكون حالة إنعزال أو عزلة بصرية (لأن الفرد

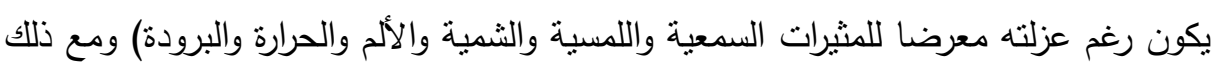

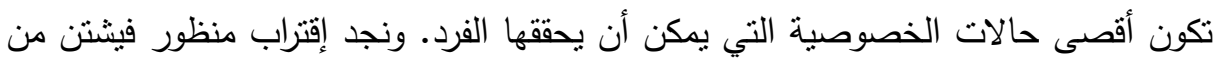
تعريفات Alexander \& Chermayeff، : "الخصوصية هي المركب المدهش للإنسحاب، لنهن الأعتماد على الذات Self-reliance، العزلة، التأمل والتفكير"، ووصف شيفي شيليز Anills الخصوصية بأنها "العلاقة الصفرية بين جماعة ما وفرد ما". 
العلاقة الحمبمية intimacy: وهي حاجة الفرد للخصوصية كعضو من جماعة ثنائية أو جماعة أكبر لتحقيق أقصى قدر ممكن من العلاقات الثخصية بين أعضاء الجماعة الثنائية

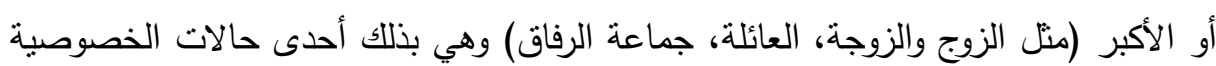

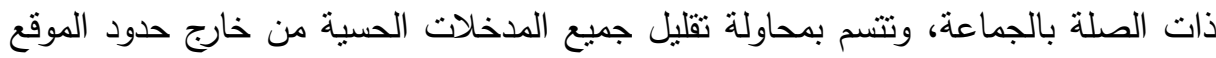
الفيزيقي المناسب. المجهولية Anonymity: فيكون الفرد مجهولاً من الآخرين وهى من إحدى حالات

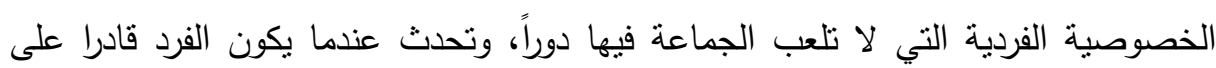

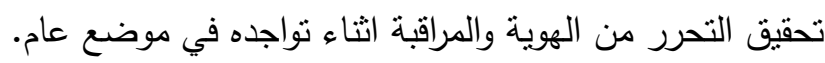
التحفظ أو التكتم Reserve: ولإشباع هذه الحالة تكمن في طبيعة العلاقات الثخصية المتبادلة أكثر من طبيعة الموضع الفيزيقي وتتظيمه، أب أنها إحدى حالات الالخصوصية فئه

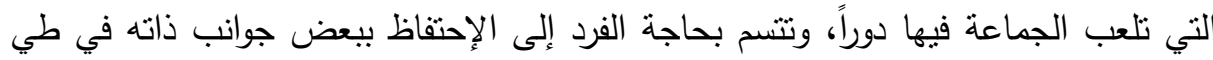
الكتمان إما لأنها شخصية جداً أو مخجلة. مجالات الحيز الثخصي: وضح العالم أدورد هيل Edward T. Hall فكرة نشوء العلاقات الإنسانية (البيئة الاجتماعية للفرد) من خلال نظرية الحيز الثخصي.

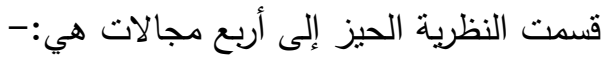

المجال الخاص: وهو الدجال الذي لا يسمح بدخوله إلا أقرب الأفراد الذين يريد الفرد لهم أن الن الني يدخلوه. كالعلاقة الطبيعية للأزواج، والأبناء، والأخوة. الدجال الشخصي: ويمنل المسافة التي يقف بها الفرد أثناء تعامله مع الآخرين الذين يعرفهه سواء كان ذللك في مكان العمل أم في الاجتماعات على اختلاف أنواعها.

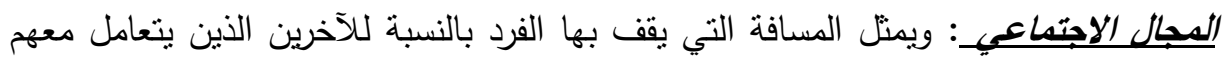

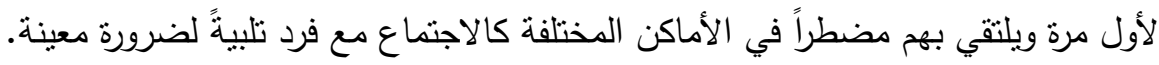

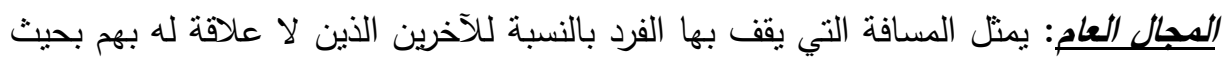
يختار المسافة التي يرتاح بها نفسياً ولا يضايق الآخرين. 
نميز بين الخصوصية والحيز الثخصي، بأنهما يمثلان حرية الفتاة فى الانفراد بذاتها

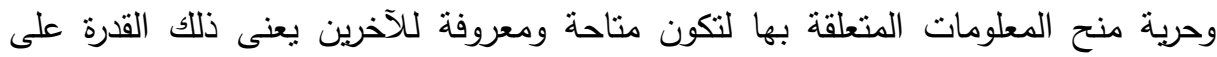
السيطرة على الحياة الخاصة لتحقيق الخصوصية، ويتحقق الحيز الثخصى الملائم للفتاة في الحصول على حدود مكانية ونفسية تعطيها الأمان فينطلب ذلك حيز مناسب على المستوى المكاني والنفسي. أدوات الدراسة: استخدم الباحثون فى هذه الدراسة أربعة مقاييس وهي: قائمة أيزنك للشخصية إعداد جابر عبد الحميد ومحد فخر الإسلام. مقياس البيئة الفيزيقة من إعداد الباحثون. مقياس الخصوصية من إعداد الباحثون. مقياس الحيز الثخصي من إعداد الباحثون. مقياس البيئة الفيزيقة: إحتوى على محورين (إستمارة بيانات أولية) ( لقياس البيئة الفيزيقية) وهدف المقياس هو الوصول لتقدير اولى عن مدى توفر الخصوصية والحيز الثخصي فى الثى لئي البيئة الفيزيقية. عدد الاسئلة (Yr) سؤال.

إجراءات الصدق والثبات: وقد تم الحصول على الصدق الظاهرى من خلال عرض المقياس على سبعة محكمين من أعضاء هيئة التدريس المتخصصين في علم النفس والطب النفسي

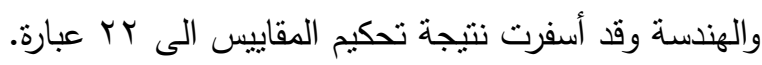
ثبات المقياس: وتم الحصول عليه عن طريق التجزئة النصفية، فقد تم تجزئة بنود مقياس

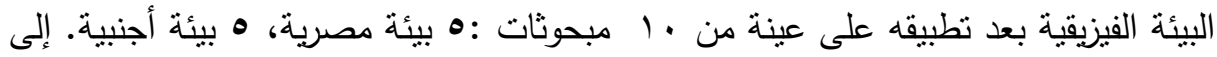
نصفين، يحتوى النصف الاول على العبارات الفردية، والنصف الثاني على العبارات الزوجية، وتم حساب الارتباط بينهما بمعامل بيرسون، حيث بلغ معامل ثبات المقياس ل111.

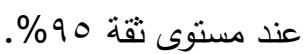
وهذا يتضح أن معامل الثبات لمقياس تحليل البيئة الفيزيقية مرضي. مقياس الخصوصية اشتمل ستة محاور تعبر عن حالات الخصوصية (الانفراد،المجولية، التحفظ،الانعزال،العلاقات الحميمية).

$$
\text { المجلد الخامس والثناثتون، الجزء الثاني، سبتمبر } 17 \text { ب r }
$$


إجراعات الصدق والثبات: وقد نم الحصول على الصدق الظاهرى من خلال عرض المقياس على على سبعة محكمين من أعضاء هيئة التدريس المتخصصين في علم الم النفس والطب

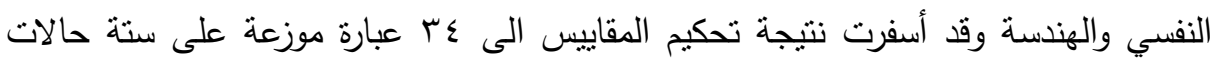

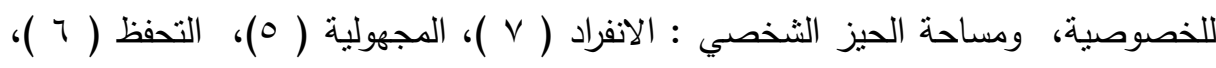

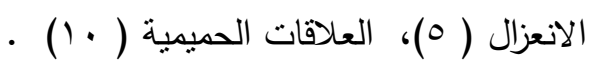

ثبات المقياس: وتم الحصول عليه عن طريق التجزئة النصفية، فقد نم تجزئة بنود مقياس

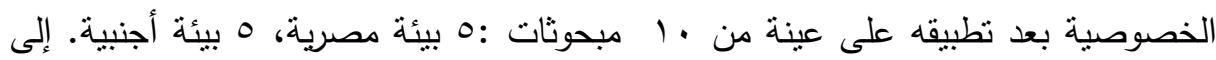
نصفين، يحتوى النصف الاول على العبارات الفردية، والنصف الثناني على العبارات الزوجية، وتم حساب الارتباط بينهما بمعامل بيرسون المقياس وحالاته لالة الارتباط

\begin{tabular}{|c|c|c|}
\hline دال عند مستوى ثقة 90\% & $\cdot, 7 \leq$ & الانفراد \\
\hline دال عند مستوى ثقة 90\% & $\cdot, 07$ & المجهولية \\
\hline دال عند مستوى ثقة $90 \%$ & $\cdot, 7 \wedge 1$ & التحفظ \\
\hline دال عند مستوى ثقة 90\% & $\cdot, 0 Y_{1}$ & الانعزال \\
\hline دال عند مستوى ثقة 90\% &., 017 & العلاقات الحميمية \\
\hline
\end{tabular}
مقياس الحيز الثخصي: احتوى على أربعة محاور رئيسية (الخاص، الثخصي، الاجتماعي، العام). (حام) إجراعات الصدق والثبات: وقد نم الحصول على الصدق الظاهرى من خلال عرض المقياس

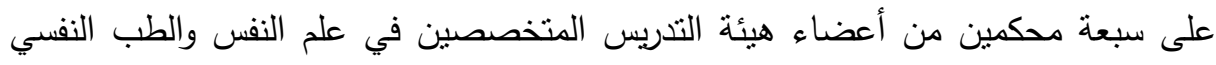
والهندسة وقد أسفرت نتيجة تحكيم المقاييس الى ل عبارة موزعة على أربعة حالات للحيز

ثبات المقياس: وتم الحصول عليه عن طريق التجزئة النصفية، فقد نم تجزئة بنود مقياس

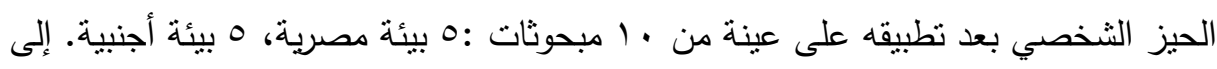


نصفين، يحتوى النصف الاول على العبارات الفردية، والنصف الثاني على العبارات الزوجية، وتم حساب الارتباط بينهما بمعامل بيرسون.

دلالة الارتباط

دال عند مستوى ثقة 90

دال عند مستوى تقة 90\%

دال عند مستوى ثقة 90\%

دال عند مستوى ثقة 90\%
معامل الارتباط

$\cdot, 7 \leq$

$\cdot, 07$

$\cdot, 7 \wedge 1$

$\cdot, 0$ Y

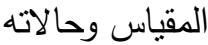

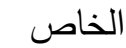

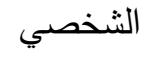

الاجتماعي

العام

وقد استخدم الباحثون المعالجات الإحصائية المتمثلة فى إختبار T للتعرف على الفروق بين العينتين واختبار كا` للاستقلالية لقياس العلاقة بين المتغيرات، ومعامل إرتباط بيرسون لتحديد لعلاقة بين أبعاد مقياس السمات النفسية وحالات مقياس الخصوصية مقياس والحيز Spss الثخصي بإستخدام برنامج التحليل الاحصائي لئين

\section{نمأئج الصراسمة}

العلاقة بين البيئة الفيزيقية ونقص الخصوصية

جدول رقم(1 ): إختبار كا' للعلاقة بين الخصوصية والبيئة الفيزيقية

\begin{tabular}{|c|c|c|c|c|}
\hline \multicolumn{2}{|c|}{ البيئة الاجنبية } & \multicolumn{2}{|c|}{ البيئةً المصرية } & \multirow{2}{*}{ الخصوصية في البيئة الفيزيقية } \\
\hline exقالتوقع & العدد & ex التوقع & العدد & \\
\hline $11, \mathrm{~V}$ & $\pi$ & $11, \mathrm{~V}$ & Ir & عالية \\
\hline $11, V$ & Ir & $11, V$ & 10 & متوسطة \\
\hline $11, \mathrm{~V}$ & 1. & $11, \mathrm{~V}$ & $\Lambda$ & منخفضة \\
\hline \multicolumn{2}{|c|}{$\cdot, \varepsilon \ldots{ }^{a}$} & \multicolumn{2}{|c|}{$r, \| 1 \varepsilon^{a}$} & قيمة كا \\
\hline \multicolumn{2}{|c|}{$r$} & \multicolumn{2}{|c|}{$\frac{r}{r<y}$} & Df \\
\hline \multicolumn{2}{|c|}{$\cdot, \wedge 19$} & \multicolumn{2}{|c|}{$\cdot r \leq V$} & مستوى الدلالة \\
\hline
\end{tabular}

يتضح من الجدول انه لا يوجد اختلاف فى توفير الخصوصية فى البيئتين العربية

والاجنبية. 


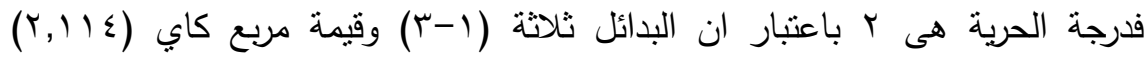
واحتمال المعنوية هي قيمة اكبر 0., • مما يعنى عدم وجود اختلاف بين البيئتين فى توفير

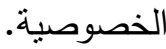

يعنى ذلك أن البيئة الفيزيقية نوفر الخصوصية فى بيئتى الدراسة، وأنه يتضح صحة الفرض القائل بوجود علاقة بين نقص الخصوصية والحيز الثخصي في البيئة الفيزيقية

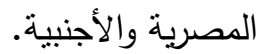

نلاحظ أن الحيز الثخصي الفيزيقى متقارب لدى عينتى الدراسة مما يدل على تمتع البيئتين بمستويات صحية من الخصوصية فى البيئة الفيزيقة للفتيات .يعكس التصميم الفيزيقي للمنزل عوامل مختلفة كثيرة منل المناخ، والتكنولوجيا، ومصادر الثروة والقيم الثقافية

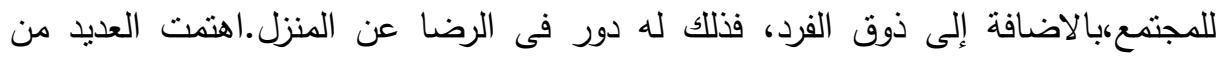

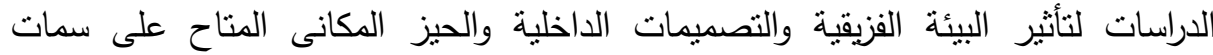

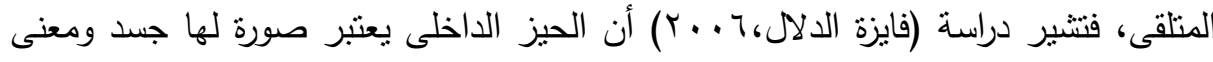
لذلك لها تأثير على المتلقى فيها، فالمعالجة البصرية للحيز المكانى تعثبر عملية إيحائية وذلك منذ العصر الحجرى حتى العصر الحديث. العلاقة بين الحيز الشخصي وسمات الشخصية:جلول(ץ): اختبار ANOVA دلالة الفروق فى درجات عينتي الدراسة على قائمة أيزنك في

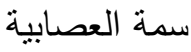

\begin{tabular}{|c|c|c|c|c|c|}
\hline \multicolumn{6}{|c|}{ ANOVA } \\
\hline \multicolumn{6}{|c|}{ العصابية } \\
\hline المعنوية & $\mathbf{F}$ & متوسط المربعات & Df & مجموع المربعات & \\
\hline$\overline{P ., \cdot Y \Lambda}$ & $0, \Gamma \cdot \varepsilon$ &., $9 \wedge 9$ & 1 &., 919 & بين المجموعات \\
\hline & & $\cdot, 1 \wedge 7$ & r & $7,10 \leqslant$ & داخل المجموعات \\
\hline & & & $\Gamma \xi$ & $V, 1 \leq r$ & المجموع \\
\hline
\end{tabular}

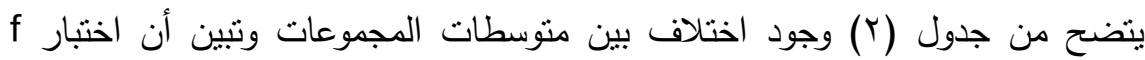

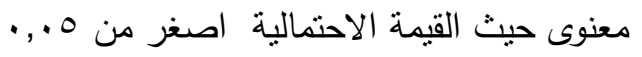
يعني ان الفروق ذات دلالة إحصائية بين عينتى الدراسة على درجة العصابية. 
وذللك يعطى دلالة من عدم التوافق فى السمات الانفعالية السلبية وكذلك السلوكية وان الفتبات عرضة لعدم الامان والاندفاع وإنخفاض نسبة العصابية ونسبة القلق والاكتئاب والعدائية والقابلية للانجراح، وترى الباحثة أن ذلك أقرب الى الصحة النفسية والسواء. جدول رقم(ץ): اختبار ANOVA دلالة الفروق فى درجات عينتي الدراسة على قائمة أيزنك

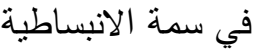

\begin{tabular}{|c|c|c|c|c|c|}
\hline \multicolumn{6}{|c|}{ ANOVA } \\
\hline \multicolumn{6}{|c|}{ الاتبساطية } \\
\hline المعنوية: & $\mathbf{F}$ & متوسط المربعات & Df & مجموع المربعات & \\
\hline$\cdot, \cdots$ & $r \wedge, Y \wedge T$ & $r, 907$ & 1 & $r, 907$ & بين المجموعات \\
\hline & & $\cdot, 1 \leqslant$. & r & $\varepsilon, 710$ & داخل المجموعات \\
\hline & & & $r \varepsilon$ & $\Lambda, 0 \vee 1$ & المجموع \\
\hline
\end{tabular}

يتضح من الجدول (r) وجود اختلاف بين متوسطات المجموعات وتبين أن اختبار معنوى حيث القيمة الاحتمالية اقل من هـ.,

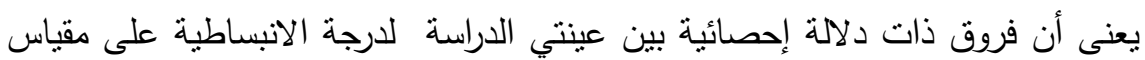
أيزنك. وتدل الدرجات العالية للانبساطية على سمات شخصية تركز على الاجتماعية والبهجة والدفء وتوكيد الذات وأنهم كثيروا الكلام والنشاط والاجتماعيات ـ فكانت الاستجابات على الى الى

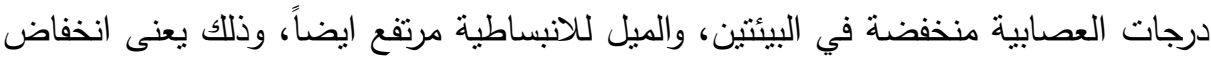
نسبة العصابية وسمات القلق والاكتئاب والعدائية والقابلية للانجراح، وتدل الدرجات العالية للانبساطية على سمات شخصية نركز على الاجتماعية والبهجة والدفه وتوكيد الذات وكثرة

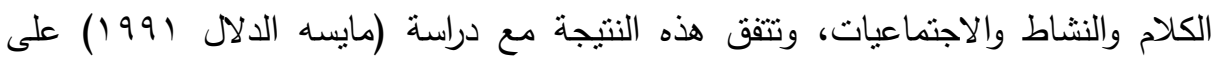
الاناث متأخرات البلوغ فوجدت أن الاناث مبكرات البلوغ أكثر انبساطيه من الاناث متأخرات

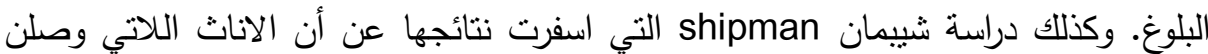

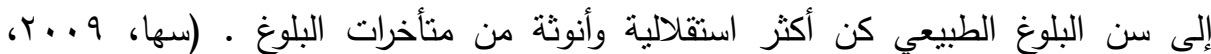

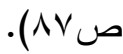


فى دراسة (سمية إسماعيل، • • ب) يتضح وجود علاقة بين متغيرات البيئة الفيزيقية

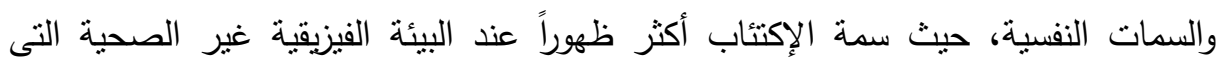
تعانى من الإزدحام وسوء حالة المسكن ونقص الخصوصية ونقص فى الخدمات والمرافق،

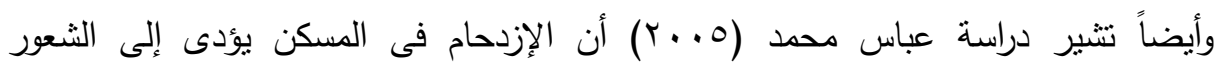

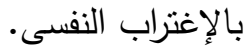

جدول(؛): يوضح دلالة الفروق فى درجات عينتي الدراسة فى تفضيل حالات الخصوصية

\begin{tabular}{|c|c|c|c|c|}
\hline \multicolumn{2}{|c|}{ العينة الاجنيية } & \multicolumn{2}{|c|}{ العينة المصريـة } & \multirow{2}{*}{ فضضيل حالات الخصوصية } \\
\hline النسبة \% \% & العدد & النسبة \% \% & العدد & \\
\hline$\%$ \% V,1 & $\overline{T V}$ & $\% \circ \vee, 1$ & $\overline{r .}$ & الانفراد \\
\hline$\% \wedge r, q$ & rq & $\% 70, \mathrm{~V}$ & Tr & المجهولية \\
\hline$\% \wedge r, q$ & rq & $\% \circ \vee, 1$ & r. & التحفظ \\
\hline$\% \vee \backslash, 乏$ & ro & $\% \uparrow 0, V$ & rr & الانعزال \\
\hline$\% \vee \varepsilon, r$ & FT & $\%$ \%\, ฯ & $T \leq$ & العلاقات الحميمية \\
\hline
\end{tabular}

يتضح من جدول (ع) ارتفاع نسبة تفضيل حالات الخصوصية فى البيئتين المصرية والاجنبية، وترى الباحثة أن تفضيل الفتيات لحالات الخصوصية تعبر عن الصحة النفسية الطبيعية للفتيات في تلك المرحلة المراهقة تحديداً. كثفت نتائج الدراسة الراهنة عن وجود فروق دالة إحصائيا بين عينتى فتيات البيئة المصرية والبيئة الاجنبية، وجاءت الفروق دالة على جميع متغيرات الخصوصية وكانت الفروق بنسبة ضئيلة تبين التجانس فى الاستجابات، وجاءت الفروق دالة فى صالح العينة الاجنبية فكانت نسبة التفضيل لايهن لحالات الخصوصية و (الانفراد، المجهولية، التحفظ، الانعزال، العلاقات الحميمه) أعلى من عينة فتيات البيئة المصرية. وترى الباحثة أن إستجابات فنتيات البيئة الأجنبية تتأثر بمتغير آخر وهو الاغنئه يتضح الميل للخصوصية ووضع حدود والالتزام بحيز شخصي أعلى من أصحاب البيئة المصرية المتواجدين في بيئتهم الاصلية الطبيعية. 
وعلى نحو آخر ، فإن دراسات عديدة أشارت إلى أن الأفراد يكونون أكثر إدراكا للبيئة

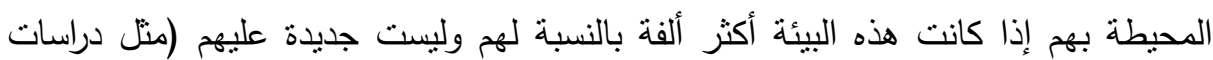
Sadalla إيفانز EVANS,1980، روبرت وآخرون, ROBERT et al، وسدالا وستابلن

$$
\text { (أحمد العتيق، ( \& Staplin }
$$

تلعب الملامح غير الفيزيقية مثل توفر الخصوصية ونمط الروابط الاجتماعية مع الجيران

$$
\text { والقيم التقافية للمجنمع دوراً في الرضا عن السكن. }
$$

قد وصف فيشتين Westin, أربع حالات مختلفة للخصوصية هي: العزلة، والمودة أو العلاقة الحميمة، والمجهولية (جهل الآخرين بشخصية الفرد)، والتحفظ أو التكتم. ولكل مظهر هيته

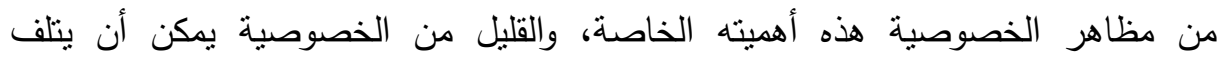
ويضعف بشكل خطير إحساس الفتيات بسلامتهاهوقدرتها لأن توظف بشكل فعال. وعلى الرغم

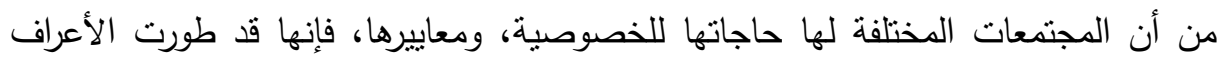

\begin{tabular}{|c|c|c|c|c|}
\hline \multicolumn{5}{|c|}{ جدول(ه): يوضح حالات الحيز الشخصي } \\
\hline \multicolumn{2}{|c|}{ العينة الاجنبية } & \multicolumn{2}{|c|}{ العينة المصرية } & \multirow{2}{*}{ حالات الحيز الثخصي } \\
\hline النسبة \% & العدد & النسبة \% & العدد & \\
\hline$\% \vee \vee v, 1$ & TV & $\% \circ \vee, 1$ & r. & 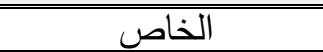 \\
\hline$\% \wedge r, q$ & rq & $\% 70, V$ & Tr & الشخصي \\
\hline$\% \wedge r, q$ & rq & $\% \circ \vee, 1$ & $r$. & الاجتماعي \\
\hline$\% \vee \backslash, \varepsilon$ & ro & $\% \curlyvee 0, V$ & $r T$ & العاد \\
\hline
\end{tabular}
والتقاليد الإجتماعية بشكل أو بآخر لتسمح للفتيات بالتحكم فى تقدير أنفسهم بطريقة ما.

يوضح الجدول رقم (0) حالات الحيز الثخصي للبيئتين، فيتبين من المقارنة أن الفتيات في البيئتين تحافظ على حيزها الشخصي وحيزها العام والاجتماعي بصورة منقاربة، وفي البيئة الاجنبية نرتفع نسبة الحفاظ على الحيز الخاص اكثر من البيئة المصرية. وللحيز الشخصي وظائف كثيرة متتوعة، فهو يخدم أو يفيد من حيث كونه منطقة محايدة تحمينا من التهديد الجسدي والنفسي، كما بساعدنا على التوافق مع المدخلات الحسية التي

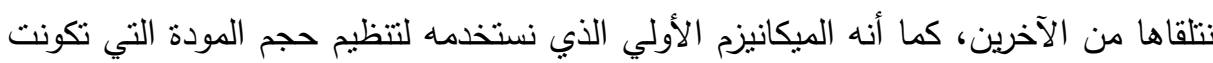

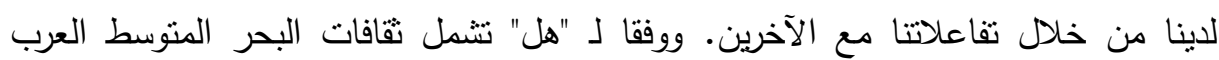
وجنوب أوروبا وأمريكا اللاتينية. ويظهر أفراد هذه المجتمعات كمية كبيرة من المودة في

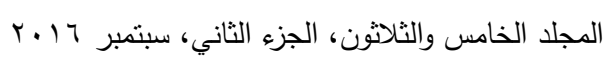


سلوكياتهم المكانية،ويستخدمون مسافات تفاعلية مثقاربة جداً، والكثير من التلامس والاتصال

$$
\text { بالعين (وذلك مع مسافات وعلاقات المودة ). }
$$

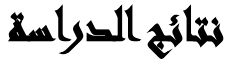

فيما يلي نوضح أهم النتائج التي توصلت لها الدراسة الراهنة:

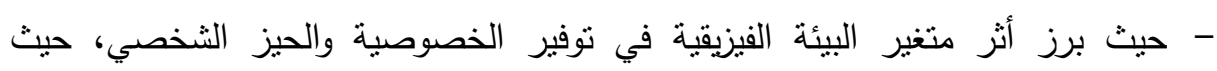
فضلت الفتيات فى البيئتين البيئة الفيزيقية الموفرة للخصوصية، لئية والحيز الشخصي.

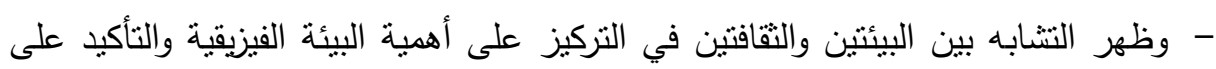
الارتباط معها من خلال عدم وجود فروق بين المجتمعين في بعد البيئة الفيزيقية. - تفضيل الإناث لمستويات عالية من الخصوصية والحيز الثخصي، وإرتباطها بتحقيق وإثباع حاجات أساسية لديها.

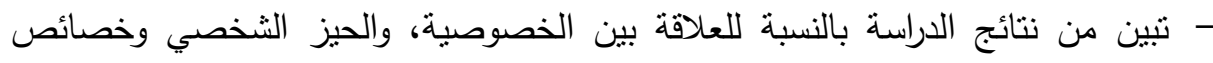
الثخصية، أن هناك علاقة إيجابية بين تفضيل أبعاد الخصوصية الفردية وتحقيق حيز

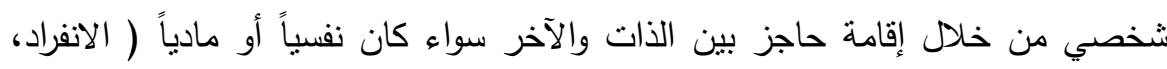
العزلة، المجهولية، التحفظ) وبين وجود خصائص شخصية غير مرغوبة إجتماعيا أو وجود نقص فى مهارات التفاعل الاجنماعي (عصابية، ذهانية، ضعف في توكيد الذات، إنطواء) وذللك فى العينتين، وهذا يدل على أن تفضيل الإناث لأبعاد الخصوصية الفردية التي

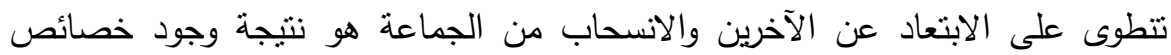

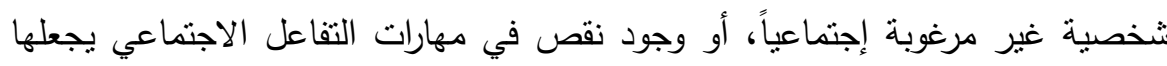

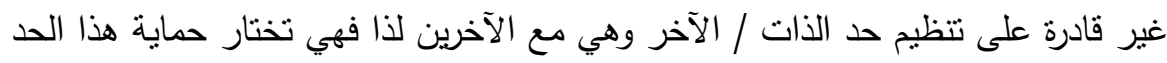

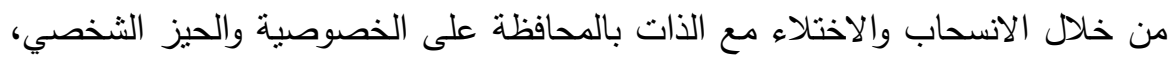
ومع وضع الإعتبار لعادات التنشئة الاجتماعية في البيئتين الذي يجعل الاتجاه للخصوصية أمر طبيعي. 


\section{توسيايت الصراسمات}

تثنير نتائج الدراسة الحالية والدراسات السابقة الى تأثير إنتهاك الخصوصية وإنتهاك

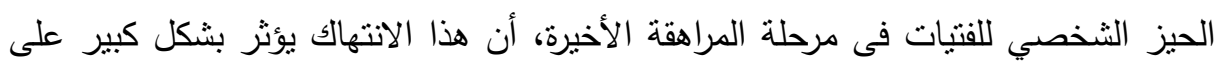
سمات الثخصية الدقسمة الى سمات الانبساطية والانطواء، ويرى الباحثون مراعاة:

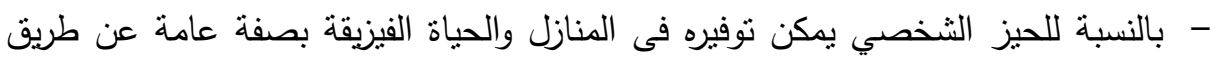
استغلال الفراغات والمساحات الغير مستغلة هنسياً.

- بالنسبة للخصوصية فتتقسم حالات الخصوصية الى خاصة وعامة والخاصة المسؤول عنها المربون والبيئات الاولية، فيوصي الباحثون بمراعاة اسس التربية التي تحافظ على الثى

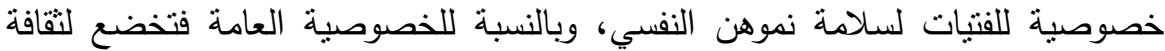
المجتمع الذي يحتاج لتوعية من المتخصصين. - يرى الباحثون بإصدار قوانين للحماية من انتهاك الخصوصية والحيز الثخصي على

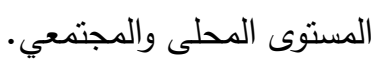

\section{المرالئ}

أحمد مصطفى العثيق( (. ب): الادراك البئي عند الاطفال - دراسة مقارنة بين الريف والحضر ، مطبوعات مركز البحوث والدراسات الاجتماعية الإنية

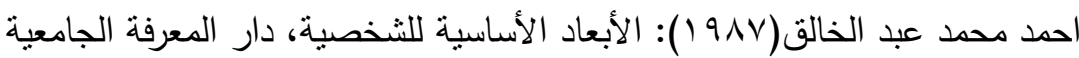

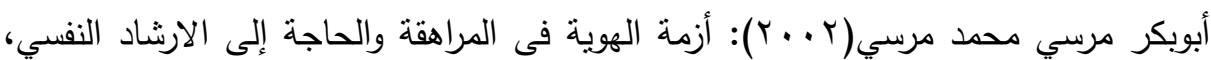

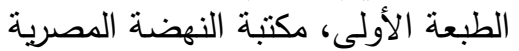

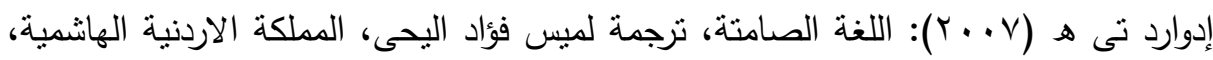
دار مولا الاهلية

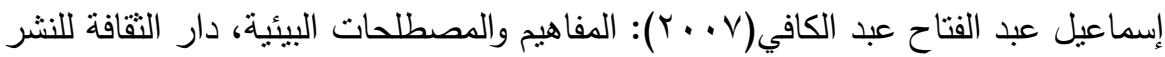

بثنينة قنديل(9V0 ()): التغير النفسى والتغير الإجتماعى فى قرية مصرية، الجمعية المصرية للاراسات النفسية، الهيئة المصرية العامة للكتاب

جابر عبد الحميد جابر وآخرون( (991)): علم النفس البيئي، مصر، دار النهضة العربية

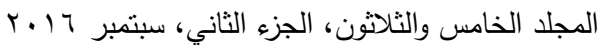




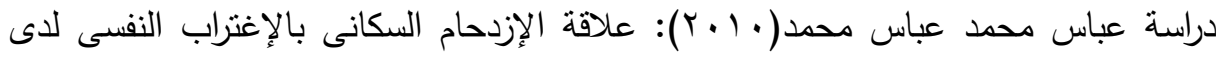
شرائح فى بيئات متباينه فى مدينة القاهرة، معهذ الدراسات البيئية، دكتوراه.

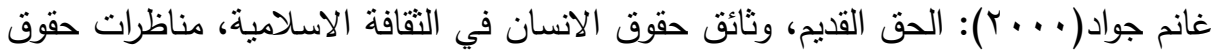
الانسان ^، الطبعة الثانية، مركز القاهرة لدراسات حقوق الانيان الانسان.

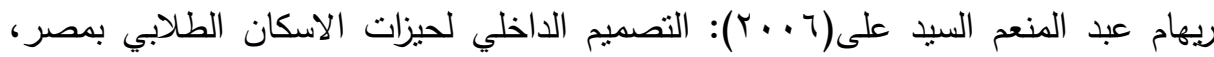
رسالة ماجستير، قسم الديكور، كلية الفنون الجميلة، جامعة الاسكندرية.

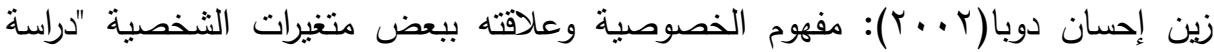
مقارنة"، رسالة ماجستير، كلية البنات للأداب والعلوم الحديثة والتربية، قسم علم

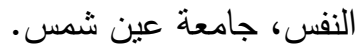

سها ابراهيم مزيد ابراهيم(9 . . ب): البلوغ وعلاقته ببعض المتغيرات النفسية الاجتماعية، رسالة

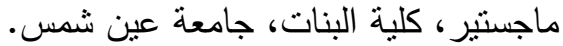

فرانسيس ت، ك آندرو ماك(991 (191): علم النفس البيئي، ترجمة عبد اللطيف محمد خليفه، جمعة سيد يوسف، مطبوعات جامعة الكويت.

لورانس أ. برافين(• ( ب): علم الثخصية، الجزء الثانى، المركز القومى للترجمة. الطبعة الأولى.

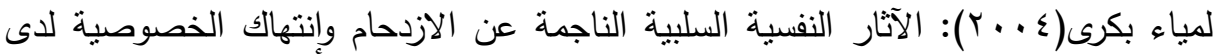
طلاب المدن الجامعية، جامعة القاهرة.

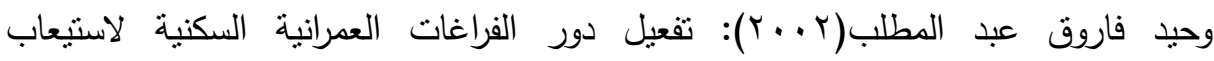
الاحتياجات الاجتماعية والنفسية للقاطنين، رسالة دكتوراة، قسم الهندسة البيئية، معهد الدرسات والبحوث البيئية، جامعة عين شمس.

Edward, T. Hall (1959): The Silent Language. A System for the national of proxemics Behavior, American Anthropologist

Kennedy, D.P.; Gläscher, J.M. and Adolph, R. (2009): Personal space regulation by the human amygdala. Nat Neurosis. 


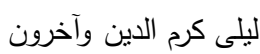

\title{
LACK OF PRIVACY AND PERSONAL SPACE AND THEIR EFFECT OVER THE PERSONALITY TRAITS OF TEENAGE GIRLS - COMPARATIVE ENVIRONMENTAL STUDY
}

Karam El Din, Laila ${ }^{(1)}$; El-Brombaly, H. H. ${ }^{(2)}$; Hany, A. F. ${ }^{(3)}$ and Lashien, Mona, A.

1) Motherhood and Childhood Institute, Ain Shams University.2) Faculty of Engineer, Ain Shams University 3) Environmental Studies and Research, Ain Shams University

\begin{abstract}
This study investigates the term "lack of privacy and personal space" for its influence on the personality traits of persons. This research aims at inquiring the existence of any correlation between the personality traits on one hand, and the lack of privacy and personal space on the other. In this regard, the research focused on teenage girls, in their third stage of adolescence, in two different environments.

The subjects of the research were: Egyptian teenage female students $(\mathrm{N}=35)$ and foreign teenage female students from East Asia countries to study at Al-Azhar university $(\mathrm{N}=35)$, both groups aged 18:20 and were assessed using A scale - measure - of privacy and personal space, a scale to analyze the physical environment (prepared by the researcher) and Eysenck personality questionnaire (translated into Arabic by Gabber Abdel-Hamyeed and Mohamed Fakhr Al-Islam). The place of the study: Cairo. The research adopted the comparative method.
\end{abstract}

$$
\text { المجلد الخامس والثلاثون، الجزء الثاني، سبتمبر } 1 \text { ا ـ ب }
$$




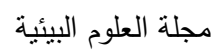

A number of results can be listed as follows: There is a functional significant relation between the lack of privacy and personal space, and the development of the personality traits of teenage female. There is a functional significant relation between the lack of privacy and personal space in one hand, and the differences in the privacy preferences between the two groups studied in this research. There is a functional significant relation between the personality traits and the lack of privacy and personal space. 